\title{
Where Do People Go for Gonorrhea and Chlamydia Tests: A Cross-sectional View of the Central Indiana population, 2003-2014
}

\author{
Teresa A. Batteiger, $\mathrm{MD}^{1}$, Brian E. Dixon, $\mathrm{PhD}^{2,3}$, Jane Wang, $\mathrm{PhD}^{2}$, Zuoyi Zhang, $\mathrm{PhD}^{2}$, \\ Guoyu Tao, $\mathrm{PhD}^{4}$, Yan Tong ${ }^{2}$, Wanzhu Tu, $\mathrm{PhD}^{1,2,3}$, Sarah A. Hoover ${ }^{2}$, Janet N. Arno, MD ${ }^{1,5}$ \\ ${ }^{1}$ Indiana University School of Medicine, Indianapolis, IN, USA \\ ${ }^{2}$ Regenstrief Institute, Indianapolis, IN, USA \\ ${ }^{3}$ Indiana University Richard M. Fairbanks School of Public Health, Indianapolis, IN, USA \\ ${ }^{4}$ Centers for Disease Control and Prevention, Atlanta, GA, USA \\ ${ }^{5}$ Marion County Public Health Department, Indianapolis, IN, USA
}

Corresponding author and alternate corresponding author:

Teresa A. Batteiger, MD

Indiana University School of Medicine

545 Barnhill Dr, Emerson Hall Room 421

Indianapolis, IN 46202

Phone: 317-274-8115 Fax: 317-274-1587

tbatteig@iu.edu

Conflict of Interest: The authors report no conflicts of interest.

\author{
Alternate: Janet N. Arno, MD \\ Indiana University School of Medicine \\ 640 Eskenazi Avenue, FOB, $1^{\text {st }}$ floor \\ Indianapolis, IN 46202 \\ Phone: 317-221-8360 Fax: 317-221-8340 \\ jarno@iu.edu
}


Funding: This work was supported by a contract from the U.S. Centers for Disease Control and Prevention [Contract number: 200-2011-42027 0003]. The views expressed in this publication are those of the authors and do not necessarily reflect the position or policy of the Centers for Disease Control and Prevention or the United States government.

Summary: Although STI clinics have been the most frequent test setting for men and outpatient clinics for women, emergency departments are increasingly a source of testing and morbidity.

Keywords: Chlamydia, Gonorrhea, Emergency Departments, Sexually Transmitted Infections, Epidemiology 


\section{ABSTRACT}

Background: Despite major efforts to control their spread, reported sexually transmitted infections (STI) are increasing. Using data from a mid-sized Midwest metropolitan area, we examined the settings in which individuals are tested for gonorrhea and chlamydia in relation to demographics and test result to determine where interventions may best be focused.

Methods: A de-identified and integrated registry, containing records from all patients tested for an STI from 2003-2014, was created by combining data from a large health information exchange and the reporting district's STI Program located in Indianapolis, IN. Individual characteristics and visit settings where gonorrhea and chlamydia testing was performed were analyzed.

Results: We identified 298,946 individuals with 1,062,369 visits where testing occurred at least once between the ages of 13 and 44 years. Females were tested significantly more often than males and received testing more often in outpatient clinics whereas males were most often tested in the STI clinic. Individuals who utilized both STI and non-STI settings were more likely to have a positive test at an STI or ED visit $(6.4 \%$ - 20.8\%) than outpatient or inpatient setting (0.0$11.3 \%)(\mathrm{p}<.0001)$. Test visits increased over the study period particularly in emergency departments, which showed a substantial increase in the number of positive test visits.

Conclusions: The most frequent testing sites remain STI clinics for men and outpatient clinics for women. Yet, emergency departments are increasingly a source of testing and morbidity. This makes them a valuable target for public health interventions that could improve care and population health. 


\section{INTRODUCTION}

Chlamydia and gonorrhea remain the first and second most frequent notifiable infectious diseases in the United States together numbering more than 2 million cases annually (1) at a lifetime cost of over $\$ 650$ million (2) and a loss of productivity greater than the cost of care for working individuals (3). However, despite all efforts, gonorrhea and chlamydia are still increasing (1) mandating development of alternative approaches. In the absence of vaccines, this means enforcing best practices for STI control and prevention. In addition to the recommendation to screen all sexual women aged $<25$ years, STI control in the US relies on local STI programs and associated STI clinics to diagnose, verify treatment and perform contact tracing. Increasingly however, STIs are diagnosed outside of publicly funded STI clinics. For example, in 2015, public STI clinics diagnosed 7\% of chlamydial infections, 12\% of gonorrhea and $20 \%$ of primary and secondary syphilis compared to 2003 when STI clinics reported $18 \%$, $30 \%$ and $40 \%$ of chlamydia, gonorrhea, and primary and secondary syphilis, respectively $(1,4)$. Although STI clinics in larger cities may diagnose a higher proportion of STIs (5), this decrease in the proportion of STI morbidity reported by STI clinics mandates evaluation of circumstances of testing. If individuals are going elsewhere for STI services, how should STI programs support best practices outside of the STI clinic, such as high-quality rapid testing, partner notification and evidenced-based behavioral interventions? Although prior studies of STI services have examined subpopulations, including STI clinic patients, women, Medicaid and large health maintenance organization populations, less is known about the general population regarding where individuals go for STI related services (6-16). 
According to the 2016 CDC Surveillance report, Indianapolis ranked $6^{\text {th }}$ in the rate of both gonorrhea and chlamydia among selected metropolitan statistical areas (MSAs) in 2016, but $34^{\text {th }}$ in population ${ }^{1}$. This national method of ranking is based on the number of reported cases/100,000 population but is influenced by reporting completeness, levels of screening, and population growth. The population of the Indianapolis-Carmel-Anderson MSA, which encompasses most of central Indiana, for example, increased $29 \%$ from $1,525,104$ in 2000 , to 1,971,060 in 2014 (US Census).

The medical record systems of the Indianapolis-Carmel-Anderson MSA have been linked to one another through the Indiana Network for Patient Care (INPC), the largest inter-organizational clinical data repository in the US. We examined chlamydia and gonorrhea testing over an elevenyear period using a comprehensive STI testing registry derived by combining information the INPC and the Central Indiana (Indiana District 5) STI program to determine the settings where individuals were tested, and the settings associated with the highest rate and absolute number of infections. Our goal was to optimize targeting of public health interventions.

\section{METHODS}

\section{Data Source}

Data from all individuals, ages 13 years and older, who had a chlamydia, gonorrhea, or syphilis test performed between January 1, 2003 and December 31, 2014 were extracted from the INPC, operated by the Indiana Health Information Exchange (17). These data were combined with data from individuals similarly tested by the Marion County Public Health Department's (MCPHD) STI Program. Extracted data were integrated into a comprehensive metropolitan STI registry (18). The INPC encompasses all major healthcare systems and laboratories in Central Indiana,

\footnotetext{
${ }^{1}$ US Census 2010: 2016 estimates: https://factfinder.census.gov/faces/tableservices/jsf/pages/productview.xhtml?src=bkmk https://www.census.gov/data/tables/time-series/dec/c2010sr-01.html Chapter 2 data
} 
except for the Veterans Administration. The MCPHD STI Program tests individuals at a full time public STI clinic and through several outreach programs in Indiana District 5 (roughly equivalent to the Indianapolis-Carmel-Anderson MSA). The resulting registry contains test information for chlamydia, gonorrhea, syphilis, HIV and pregnancy tests, visit information including type of clinic (i.e. Emergency Department (ED), outpatient, inpatient, or STI program), visit date (transformed relative to the patient's initial visit), visit year, diagnostic codes, infection status at time of visit and demographics including age, gender, race, and ethnicity. For this study, individuals with any gonorrhea or chlamydia test in the registry between the ages of 13 and 44 were examined. Syphilis was excluded from the current study because of the complexity of analysis associated with diagnosis and follow up testing. We analyzed individuals and visits rather than tests to control for tests performed at multiple anatomical sites at the same visit on a single individual. Individuals with a positive test at any anatomic site and visits associated with a positive test (regardless of anatomic site) were considered positive. Analyses of test visits and positivity over time were analyzed from 2005, when the use of nucleic acid amplification tests for both gonorrhea and chlamydia were standard across different settings. Some healthcare systems provided data that included the actual clinic site of testing (i.e. OB/GYN, Primary Care, etc.), specific visit location information was available on a subset of patients including $63.3 \%$ of females and $81.2 \%$ of males. These data are provided to give additional information about the type of outpatient clinics used for testing.

\section{Matching Algorithm}

Individuals were identified as matches in the two source datasets, the INPC and MCPHD STI program, using two probabilistic utilities. Both utilities use a value-based weight scaling 
modification of matching based on first, last, and middle names; social security numbers, date of birth, race, and gender $(19,20)$. Global Match is used as a standard in the INPC to match individuals from different medical record systems and assigns a unique identifier to matches. RecMatch was used to identify matches in the combined MCPHD STI program and INPC data (21). Program-generated matches below the threshold score from RecMatch were excluded. Records at or above the threshold score were reviewed manually and a score was chosen where no clearcut mismatches were seen in 100 records. Individuals who matched the 9 digits of the social security number alone, for example, if they were given the default social security number of 999-99-9999 were excluded (6.3\%). Of the 94,055 individuals who had at least one STI test in the MCPHD STI dataset, $73.6 \%$ matched one of the 449,371 individuals in the INPC dataset. These record pairs were merged.

\section{Statistics}

Descriptive statistics were performed to determine patient characteristics associated with presenting to a specific testing setting. Hispanic origin was recorded as race in the INPC but as ethnicity in the MCPHD data, with race usually designated as "other". For this reason, Hispanic and other race were combined for the analysis. According to the US Census, only $3.9 \%$ of Indianapolis residents were not white, black or Hispanic so it is likely the majority of "other" is equal to Hispanic. Characteristics of individuals presenting to various testing settings were analyzed using mean and standard deviation for continuous variables (age and average number of visits/year), and frequency or proportion for categorical variables. Comparisons among the testing settings were performed using ANOVA for continuous variables and Pearson $\boldsymbol{X}^{2}$ test for categorical variables. Characteristics of patients associated with a positive gonorrhea or chlamydia test by setting were stratified by gender and were presented using mean and standard 
deviation for continuous variables, and frequency and proportion for categorical variables. The association between patient characteristics and test setting site was evaluated by multinomial logistic regression using the generalized logit link, where the test setting site is the response variable with STI clinic as the reference. In the model, there are 4 levels (ED, inpatient, outpatient and STI clinic) in the response variable. The grouping age (13-29 and $\geq 30)$, gender and race are the predictor variables in the model, where the interaction of gender and race was included in the model to evaluate the association between the testing sites and the race in female and male, respectively. The patient effect among the testing sites was also accounted for in the model. All statistical analyses were performed using SAS version 9.4 (SAS Institute Cary, NC). The study received approval by the Institutional Review Board at Indiana University (Study No. 1311659626).

\section{RESULTS}

From the 474,201 individuals in the combined data, we identified 298,946 unique individuals, ages 13 to 44 years, who had at least one test for gonorrhea or chlamydia (referred to as an STI test) and at least one visit with encounter information in the combined dataset. These individuals had 1,062,369 visit records associated with STI testing. Most were female (79\%). Forty-three percent were white, $39 \%$ black and $14 \%$ other race.

Table 1 illustrates the first-visit demographics of the cohort according to whether they received testing only in non-STI clinic settings, such as outpatient clinics or an ED; only at the STI clinic; or both settings. Most individuals, 238,251 (79.7\%), were tested only in non-STI settings and $36,397(12.2 \%)$ were tested only in the STI clinic. A small proportion of individuals, 24,298 (8.1\%), utilized both non-STI and STI settings. These individuals were significantly younger than those who attended non-STI settings or the STI clinic exclusively. 
Males and females differed in their utilization of different settings for STI testing $(\mathrm{p}<0.0001)$. Females were more likely to go to only non-STI sites (89.2\%) than only STI sites (3.6\%) ( $p<.0001)$ for testing. Males were slightly more likely to go to only non-STI sites $(44.7 \%)$ than only STI sites $(43.6 \%)(\mathrm{p}=0.0049)$.

All races were tested most frequently in non-STI settings. Among those that utilized both STI and non-STI settings, $69.5 \%$ were black, $21.6 \%$ were white and $9 \%$ were of other or unknown race, roughly similar to the racial breakdown for gonorrhea in 2014 but disproportionate to the racial breakdown of the city of Indianapolis estimated for 2014 from the US Census which was $62 \%$ white, $28 \%$ black: The average number of visits per year was highest (2.0) among those who utilized both STI and non-STI settings for their testing, compared with 1.7 for non-STI settings only versus 1.2 for STI settings only (both $\mathrm{p}<.0001$ ).

The STI clinic and some healthcare systems provided specific patient visit location information accounting for $63.3 \%$ of female and $81.2 \%$ of male visits. In addition to the STI clinic, identified clinics included OB-GYN, primary care, and specialty care such as urology or infectious disease. In this subset, OB-GYN clinics accounted for $37.4 \%$ of female visits associated with STI testing, EDs, 27.4\%, and primary care, $16.3 \%$ ( $\mathrm{p}<.0001)$. In contrast, most males were tested in the STI clinic (61.7\%) or the ED (19.1\%). Outpatient clinics with the most male test visits were primary care (7.6\%) and OB-GYN clinics (5.6\%).

Table 2 summarizes patient characteristics for visits associated with STI testing and the percentage of visits with positive tests in each setting. Females were more likely than males to have STI test visits in all settings except the STI clinic. In addition, to gender, the setting in which individuals were tested also differed by race. Relative to the STI clinic, white females were more likely to test in the ED, inpatient setting and outpatient settings than black females. 
Similarly, compared to black males, white males were more likely to test in the ED and inpatient settings. Regardless of race, visits to the ED and the STI clinic had the highest positivity rates for both males and females, whereas outpatient and inpatient positivity rates were relatively low. Additionally, male positivity rate was higher than that for females in all settings.

To determine whether test result differed by setting among individuals with visits to both STI and non-STI settings, we examined the distribution of 203,170 visits and percent positivity of visits among 16,816 females and 7,482 males who had at least one visit to the STI clinic and at least one visit to a non-STI setting. As seen in analyses of the unselected group, the outpatient test setting was most common for all ages and females, whereas the STI clinic was the most common test setting among males. Like the unselected group, the percent positivity was highest in the ED and STI clinic settings. For females, the percent positivity was $11.5 \%$ and $14.1 \%$ for chlamydia and $6.4 \%$ and $7.9 \%$ for gonorrhea in the ED and the STI clinic respectively compared to $9.3 \%$ for chlamydia and $3.8 \%$ for gonorrhea in the outpatient setting. For males, the percent positivity was $17.5 \%$ and $17.3 \%$ for chlamydia and $19.1 \%$ and $12.6 \%$ for gonorrhea, in the ED and the STI clinic respectively compared to $15.6 \%$ for chlamydia and $10.4 \%$ for gonorrhea in outpatient setting. For those 13-29 years, the percent positivity was $14.5 \%$ and $18.7 \%$ for chlamydia and $9.4 \%$ and $11.3 \%$ for gonorrhea in the ED and STI clinic respectively compared to $11.6 \%$ for chlamydia and $5.0 \%$ for gonorrhea in the outpatient setting.

Figure 1 shows the total number of visits and distribution of total positive test visits associated with STI testing in the outpatient, ED and STI clinic settings from 2005 to 2014 for females and males. During this period, the total number of test visits increased from 86,534 to 119,149 for females and from 10,297 to 20,727 for males. Increases occurred in most but not all individual institutions including the STI clinic (data not shown). Female test visits were most frequent in 
the outpatient setting followed by the ED. The outpatient setting contributed the highest proportion of positive test visits for females, but this contribution decreased over time. In contrast, the contribution of positive test visits by the ED increased. STI clinic test visits remained relatively low for females during the study period.

The STI clinic was the most common visit setting for testing for males until 2014 when the outpatient setting surpassed it. Although the proportion of visits associated with a positive test decreased for the STI clinic throughout the study period, it remained the major source of STI morbidity for gonorrhea and chlamydia. In contrast, however, the ED became the second most common source of morbidity for males beginning in 2008 despite the increase in testing in outpatient settings. Inclusion of additional sites to the database did not account for the increase in the number of STI test visits across all sites.

\section{DISCUSSION}

This study is unique in its examination of testing across a wide variety of providers including the public health department's STI clinic. Creation of the STI registry from a large health information exchange and the public STI program was possible only because of collaboration across the disciplines of epidemiology, informatics, infectious diseases, and biostatistics $(18,22)$. Systematically captured, integrated, and normalized data available from multiple settings allowed for a more complete assessment of where individuals received chlamydia and gonorrhea testing and setting-specific positivity rates. Based on our knowledge, this type of assessment at the national level is limited, because the availability of existing data sources and the challenging management of data across multiple data sources. 
The resulting data show that the total number of visits associated with gonorrhea and chlamydia testing increased significantly during the study period, particularly after 2007 . The increase was seen in all test settings and within individual institutions. The change was greater than the increase in the region's population. Therefore, the explanation for increased test visits is likely to be multifactorial including the increased adoption of urine and self-administered vaginal swabs for testing. The increased availability of STI testing in outpatient settings provides opportunities for interventions for both men and women, such as offering PrEP to HIV negative young men who have sex with men.

Gender was a powerful determinant of where individuals were diagnosed with an STI. Consistent with surveillance reports (1) our data show the number of females with positive tests (morbidity) was greatest from non-STI settings, particularly in outpatient clinics, although the positivity rate was lowest in those settings. This is not surprising given the recommended testing guidelines for chlamydia and gonorrhea in pregnant women and women aged $\leq 24$ years (23). In contrast, the settings with the highest positivity rate were the STI clinic and ED for both females and males. The STI clinic contributed most to male STI morbidity as noted previously (7) but its contribution to morbidity is decreasing. In contrast, EDs are contributing increasingly to morbidity, in agreement with the observations of others $(12,13,16,24)$. Even among individuals in our cohort who utilized both STI and non-STI settings, visits to the STI clinic or $\mathrm{ED}$, were more likely when there was a positive test consistent with the hypothesis that even if a patient has access to an outpatient setting, they utilize settings in which they can obtain immediate services for symptoms or suspected exposures. The registry does not include fields derived from text notes which might demonstrate this type of association. Therefore, whether increased ED visits for STI testing is related to access to immediate care, increased geographic 
distribution of ED relative to STI clinics, decreased stigma (when visiting a location not dedicated to STI care) or public health services infrastructure (24) requires further investigation. ED-based STI care may not include the comprehensive services critical to STI control (6). Optimally restructuring local STI control programs to support STI care in EDs where cost may be higher (25) needs further study. Deployment of existing resources to support EDs such as a trained STI clinician in a non-acute area of the ED, assigning DIS for onsite interviews and providing ED testing results might prove to be cost effective. As changes in healthcare unfold, the impact of healthcare coverage is likely to change the distribution of sites as well (26). For this study, changes associated with the Affordable Care Act are unlikely to have played a role in where individuals went for STI care as Indiana's plan for expanded Medicaid was approved on $1 / 27 / 2015$. $^{2}$ Future updates to the Registry may reveal changes in testing behavior as a result. Although younger individuals were more likely to be tested in the outpatient setting or ED, the effect was small possibly because the outpatient settings where individuals accessed care was not known. For example, adolescents may have been tested at specialized adolescent or Family Planning clinics.

There are several limitations to note. First, the study was retrospective involving secondary data derived from electronic health records. Data was missing or incomplete particularly in types of outpatient clinics where chlamydia or gonorrhea tests occurred. While virtually all large healthcare and laboratory systems in central Indiana are included in the testing dataset, some are not. These include a small number of non-STI clinics whose tests are performed by the public health laboratory. These gonorrhea and chlamydia tests have only recently been added to the INPC. Individuals who chose to test online or mail-in tests that went to other states were not

\footnotetext{
${ }^{2}$ https://www.medicaid.gov/Medicaid-CHIP-Program-Information/ByTopics/Waivers/1115/downloads/in/Healthy-Indiana-Plan-2/in-healthy-indiana-plan-support-20-appvl01272015.pdf
} 
captured. Second, because different medical record systems used different unique identification numbers, the public health and private health system data had to be matched using probabilistic techniques. It is possible but unlikely that data from matched individuals who were truly different were merged. If this were to be the case, the impact on our findings would likely be small.

In conclusion, morbidity reports based only on number of cases must be interpreted with caution because of substantial increases in testing over the past decade. This study provides an important and feasible approach to assess time trends of where individuals received STI testing across multiple settings, and of setting-specific positivity. The results from this study provided useful information for local STI control and prevention. Additionally, these findings may be useful for other public health and medical communities in the United States. While STI clinics remain critical for the diagnosis of gonorrhea and chlamydial infections, especially in males, visits to the ED are increasing not only in number, but also in their rate of positivity presenting a unique opportunity to intervene in disease transmission. 


\section{REFERENCES}

1. Centers for Disease Control and Prevention. Sexually Transmitted Disease Surveillance 2015. Atlanta, GA: U.S. Department of Health and Human Services; 2016.

2. Owusu-Edusei K, Jr., Chesson HW, Gift TL, et al. The estimated direct medical cost of selected sexually transmitted infections in the United States, 2008. Sex Transm Dis. 2013;40(3):197-201.

3. Owusu-Edusei K, Jr., Roby TM, Chesson HW, Gift TL. Productivity costs of nonviral sexually transmissible infections among patients who miss work to seek medical care: evidence from claims data. Sex Health. 2013;10(5):434-7.

4. Centers for Disease Control and Prevention. Sexually Transmitted Disease Surveillance 2003. Atlanta, GA: U.S. Department of Health and Human Services. 2004.

5. Golden MR, Kerndt PR. Improving clinical operations: can we and should we save our STD clinics? Sex Transm Dis. 2010;37(4):264-5.

6. Sequeira S, Morgan JR, Fagan M, Hsu KK, Drainoni ML. Evaluating Quality of Care for Sexually Transmitted Infections in Different Clinical Settings. Sex Transm Dis. 2015;42(12):717-24.

7. Hoover KW, Parsell BW, Leichliter JS, et al. Continuing Need for Sexually Transmitted Disease Clinics After the Affordable Care Act. Am J Public Health. 2015;105 Suppl 5:S690-5.

8. Pathela P, Klingler EJ, Guerry SL, et al. Sexually transmitted infection clinics as safety net providers: exploring the role of categorical sexually transmitted infection clinics in an era of health care reform. Sex Transm Dis. 2015;42(5):286-93. 
9. Cramer R, Leichliter JS, Gift TL. Are safety net sexually transmitted disease clinical and preventive services still needed in a changing health care system? Sex Transm Dis. 2014;41(10):628-30.

10. Hogben M, Bloom F, McFarlane M, St Lawrence JS, Malotte CK, Group GS. Factors associated with sexually transmitted disease clinic attendance. Int $\mathbf{J}$ Nurs Stud. 2004;41(8):911-20.

11. Leichliter JS, Copen C, Dittus PJ. Confidentiality Issues and Use of Sexually Transmitted Disease Services Among Sexually Experienced Persons Aged 15-25 Years - United States, 2013-2015. MMWR Morb Mortal Wkly Rep. 2017;66(9):237-41.

12. Frost JJ. Trends in US women's use of sexual and reproductive health care services, 1995-2002. Am J Public Health. 2008;98(10):1814-7.

13. Haderxhanaj LT, Gift TL, Loosier PS, Cramer RC, Leichliter JS. Trends in receipt of sexually transmitted disease services among women 15 to 44 years old in the United States, 2002 to 2006-2010. Sex Transm Dis. 2014;41(1):67-73.

14. Lawrence JM, Zenilman J, Kamb ML, et al. Sources of recent sexually transmitted disease (STD)-related health care for STD clinic patients. Sex Transm Dis. 2001;28(9):535-8.

15. Brackbill RM, Sternberg MR, Fishbein M. Where do people go for treatment of sexually transmitted diseases? Fam Plann Perspect. 1999;31(1):10-5.

16. Drainoni ML, Sullivan M, Sequeira S, Bacic J, Hsu K. Health reform and shifts in funding for sexually transmitted infection services. Sex Transm Dis. 2014;41(7):455-60. 
17. Overhage J. The Indiana Health Information Exchange. In: Dixon B, ed. Health Information Exchange: Navigating and Managing a Network of Health Information Systems. 1 ed. Waltham, MA: Academic Press; 2016.

18. Dixon BE, Tao G, Wang J, et al. An Integrated Surveillance System to Examine Testing, Services, and Outcomes for Sexually Transmitted Diseases. Stud Health Technol Inform. 2017;245:361-5.

19. McFarlane T, Dixon B, Grannis S. Client Registries: Identifying and Linking Patients. In: Dixon B, ed. Health Information Exchange: Navigating and Managing a Network of Health Information Systems. 1 ed. Waltham, MA: Academic Press; 2016:163-82.

20. Aldridge RW, Shaji K, Hayward AC, Abubakar I. Accuracy of Probabilistic Linkage Using the Enhanced Matching System for Public Health and Epidemiological Studies. PLoS One. 2015;10(8):e0136179.

21. Grannis SJ, Overhage JM, Hui S, McDonald CJ. Analysis of a probabilistic record linkage technique without human review. AMIA Annu Symp Proc. 2003:259-63.

22. Dixon B. What is Health Information Exchange? In: Dixon B, ed. Health Information Exchange: Navigating and Managing a Network of Health Information Systems. 1 ed. Waltham, MA: Academic Press; 2016:3-20.

23. Workowski KA. Centers for Disease Control and Prevention Sexually Transmitted Diseases Treatment Guidelines. Clin Infect Dis. 2015;61 Suppl 8:S759-62.

24. Pearson WS, Peterman TA, Gift TL. An increase in sexually transmitted infections seen in US emergency departments. Prev Med. 2017;100:143-4. 
25. Owusu-Edusei K, Patel CG, Gift TL. Does place of service matter? A utilisation and cost analysis of sexually transmissible infection testing from 2012 claims data. Sex Health. 2016;13(2):131-9.

26. Mikati T, Maloney P, Tabidze I, Mehta SD. The Change in Insurance Status Among Patients Seeking Care at Chicago Sexually Transmitted Disease Clinics After Affordable Care Act Implementation. Sex Transm Dis. 2016;43(4):260-3. 


\section{Figure Legends}

Figure 1: Distribution of the total number of visits and proportion of positive visits by setting from 2005-2014 
Figure 1
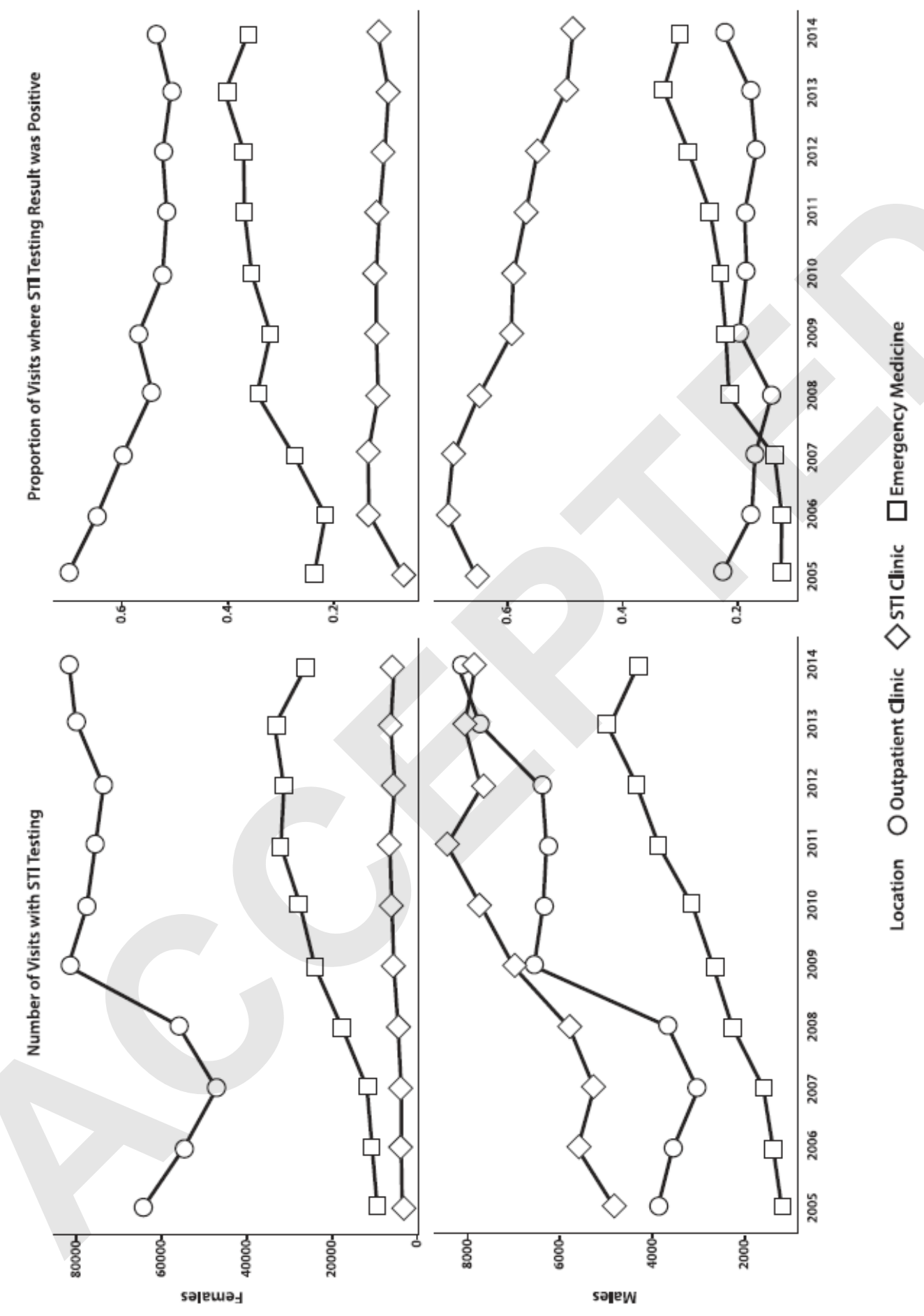
Table 1. Patient Characteristics by Testing Setting: Non-STI vs. STI vs. Both

\begin{tabular}{|c|c|c|c|c|c|c|}
\hline Demographics & Subgroup & $\begin{array}{c}\text { Total } \\
(298,946)\end{array}$ & $\begin{array}{c}\text { Non-STI } \\
(\mathrm{N}=\mathbf{2 3 8 , 2 5 1 )}\end{array}$ & $\begin{array}{c}\text { STI } \\
(\mathrm{N}=\mathbf{3 6 , 3 9 7 )}\end{array}$ & $\begin{array}{c}\text { Both } \\
(\mathrm{N}=\mathbf{2 4 , 2 9 8 )}\end{array}$ & p-value \\
\hline Age Mean(SD) & & $26.03 \pm 7.47$ & $26 \pm 7$ & $28 \pm 7$ & $24 \pm 7$ & $<.0001$ \\
\hline \multirow{2}{*}{ Gender } & Female & 235,163 & $209,767(89.2 \%)$ & $8,580(3.6 \%)$ & $16,816(7.2 \%)$ & \multirow{2}{*}{$<.0001$} \\
\hline & Male & 63,783 & $28,484(44.7 \%)$ & $27,817(43.6 \%)$ & $7,482(11.7 \%)$ & \\
\hline \multirow{4}{*}{ Race } & $\overline{\text { Black }}$ & 115,448 & $81,414(34.2 \%)$ & $17,151(47.1 \%)$ & $16,883(69.5 \%)$ & \multirow{4}{*}{$<.0001$} \\
\hline & Other & 41,688 & $37,409(15.7 \%)$ & $2,584(7.1 \%)$ & $1,695(7 \%)$ & \\
\hline & Unknown & 13,810 & $12,483(5.2 \%)$ & $852(2.3 \%)$ & $475(2 \%)$ & \\
\hline & White & 128,000 & $106,945(44.9 \%)$ & $15,810(43.4 \%)$ & $5,245(21.6 \%)$ & \\
\hline$\overline{\text { Average }}$ & & \multirow{3}{*}{$1.68 \pm 1.37$} & \multirow{3}{*}{$1.66 \pm 1.35$} & \multirow{3}{*}{$1.23 \pm 0.56$} & \multirow{3}{*}{$2.00 \pm 1.70$} & \multirow{3}{*}{$<.0001$} \\
\hline $\begin{array}{l}\text { number of } \\
\text { visits/year }\end{array}$ & & & & & & \\
\hline Mean \pm SD & & & & & & \\
\hline
\end{tabular}

Table 1: In this Table, patient age is that at first visit by site. In the case of the Both category, the age at first visit to any site was recorded. Comparisons among the testing settings were performed using ANOVA for continuous variables and Pearson $\boldsymbol{X}^{\mathbf{2}}$ test for categorical variables. 
Table 2. Multinomial logistic regression of Visit Setting with a Positive STI Test (Gonorrhea and/or Chlamydia) in relation to Age, Gender, and Race by Gender

\begin{tabular}{|c|c|c|c|c|c|c|c|c|c|}
\hline & & \multicolumn{3}{|c|}{$E D$} & \multicolumn{3}{|c|}{ Outpatient } & \multicolumn{2}{|c|}{$\begin{array}{l}\text { STI Clinic } \\
\text { (reference) }\end{array}$} \\
\hline Characteristic & & $\mathrm{N}$ & $\begin{array}{c}\text { Positivity } \\
\text { (\%) }\end{array}$ & $\begin{array}{c}\text { OR } \\
(95 \% \mathrm{CI})\end{array}$ & $\mathrm{N}$ & $\begin{array}{c}\text { Positivity } \\
(\%)\end{array}$ & $\begin{array}{c}\text { OR } \\
(95 \% \mathrm{CI})\end{array}$ & $\mathrm{N}$ & $\begin{array}{c}\text { Positivity } \\
(\%)\end{array}$ \\
\hline \multirow[t]{2}{*}{ Age } & $\begin{array}{l}13-29 \\
\text { years }\end{array}$ & 183,407 & $15.9 \%$ & $\begin{array}{c}1.43 \\
(1.39,1.48)\end{array}$ & 472,485 & $10.0 \%$ & $\begin{array}{c}1.2 \\
(1.17,1.24)\end{array}$ & 78,169 & $25.90 \%$ \\
\hline & $\geq 30$ years & 62,135 & $7.4 \%$ & reference & 191,289 & $3.4 \%$ & reference & 40,361 & $14.20 \%$ \\
\hline \multirow[t]{2}{*}{ Gender } & Female & 218,506 & $12.4 \%$ & $\begin{array}{c}10.01 \\
(9.54,10.52)\end{array}$ & 620,965 & $7.8 \%$ & $\begin{array}{c}19.55 \\
(18.59,20.57)\end{array}$ & 45,814 & $18.4 \%$ \\
\hline & Male & 27,036 & $24.8 \%$ & reference & 42,809 & $13.4 \%$ & reference & 72,716 & $24.1 \%$ \\
\hline \multirow{4}{*}{ Race: Female } & Black & 100,952 & $15.5 \%$ & $\begin{array}{c}0.57 \\
(0.55,0.59)\end{array}$ & 333,249 & $10.2 \%$ & $\begin{array}{c}0.96 \\
(0.93,1.00)\end{array}$ & 27,627 & $19.4 \%$ \\
\hline & Other & 25,314 & $10.5 \%$ & $\begin{array}{c}1.13 \\
(1.06,1.22)\end{array}$ & 85,343 & $4.8 \%$ & $\begin{array}{c}1.95 \\
(1.82,2.08)\end{array}$ & 3,509 & $18.2 \%$ \\
\hline & Unknown & 5,383 & $11.6 \%$ & $\begin{array}{c}0.79 \\
(0.71,0.88)\end{array}$ & 32,230 & $4.2 \%$ & $\begin{array}{c}2.39 \\
(2.16,2.64)\end{array}$ & 1,093 & $16.2 \%$ \\
\hline & White & 86,857 & $9.4 \%$ & reference & 170,143 & $5.2 \%$ & reference & 13,585 & $16.6 \%$ \\
\hline \multirow{4}{*}{ Race: Male } & Black & 13,916 & $31.2 \%$ & $\begin{array}{c}0.7 \\
(0.67,0.74)\end{array}$ & 24,071 & $17.4 \%$ & $\begin{array}{c}1.05 \\
(1.00,1.1)\end{array}$ & 72,716 & $28.3 \%$ \\
\hline & Other & 2,452 & $20.9 \%$ & $\begin{array}{c}1.35 \\
(1.24,1.47)\end{array}$ & 4,706 & $8.9 \%$ & $\begin{array}{c}2.24 \\
(2.05,2.45)\end{array}$ & 45,665 & $(19.4 \%)$ \\
\hline & Unknown & 1,389 & $28.1 \%$ & $\begin{array}{c}2.43 \\
(2.14,2.76)\end{array}$ & 3,280 & $6.5 \%$ & $\begin{array}{c}4.96 \\
(4.30,5.71)\end{array}$ & 4,200 & $25.6 \%$ \\
\hline & White & 9,279 & $15.7 \%$ & reference & 10,752 & $8.4 \%$ & reference & 1,323 & $16.1 \%$ \\
\hline
\end{tabular}


Table 2: All patient visits were included in this table. Positivity percentage included positive tests for gonorrhea and/or chlamydia. The STI clinic was used as the reference setting for each subgroup.

Individuals $\geq 30$ years were used as a reference when comparing test setting by age. Males were used as the reference when comparing the test setting between genders. White race was used as the reference when comparing the setting between races within gender. This analysis allows odds ratios (ORs) to be compared across 2 variables (e.g. setting and age). For example, individuals 13-29 were 1.43 times as likely to receive a test in the ED as older individuals were to receive a test at the STI clinic. Also, a larger proportion of individuals receiving tests in the ED were 13-29 years than the outpatient setting or STI clinic. Inpatient visits are not shown because the number of tests done in this setting was small. 